\title{
Investigation for a multi-silique trait in Brassica napus by alternative splicing analysis
}

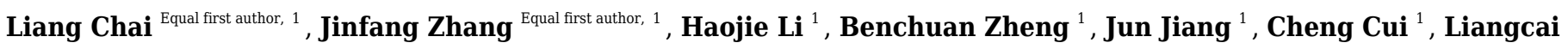 \\ Jiang ${ }^{\text {Corresp. } 1}$ \\ ${ }^{1}$ Sichuan Academy of Agricultural Sciences, Crop Research Institute, Chengdu, Sichuan Province, China \\ Corresponding Author: Liangcai Jiang \\ Email address: jlcrape@163.com
}

Background. Flower and fruit development are vital stages of the angiosperm lifecycle. We previously investigated the multi-silique trait in the rapeseed (Brassica napus) line zws-ms on a genomic and transcriptomic level, leading to the identification of two genomic regions and several candidate genes associated with this trait. However, some events on transcriptome level, like alternative splicing, were poorly understood.

Methods. Plants from zws-ms and its near-isogenic line (NIL) zws-217 were both grown in Xindu with normal conditions and a colder area Ma'erkang. Buds from the two lines were sampled and RNA was isolated to perform the transcriptomic sequencing. The numbers and types of alternative splicing (AS) events from the two lines were counted and classified. Genes with AS events and expressed differentially between the two lines, as well as genes with AS events which occurred in only one line were emphasized. Their annotations were further studied.

Results. From the plants in Xindu District, an average of 205,496 AS events, which could be sorted into 5 AS types, were identified. zws-ms and zws-217 shared highly similar ratios of each AS type: The alternative 5' and 3' splice site types were the most common, while the exon skipping type was observed least often. Eleven differentially expressed AS genes were identified, of which four were upregulated and seven were downregulated in zws-ms. Their annotations implied that five of these genes were directly associated with the multi-silique trait. While samples from colder area Ma'erkang generated generally reduced number of each type of AS events except the Intron Retention; but the number of differentially expressed AS genes increased significantly. Further analysis found that among the 11 differentially expressed AS genes from Xindu, three of them maintained the same expression models, while the other 8 genes did not show significant difference between the two lines in expression level. Additionally, the 205 line-specifically expressed AS genes were analyzed, of which 187 could be annotated, and two were considered to be important.

Discussion. This study provides new insights into the molecular mechanism of the agronomically important multi-silique trait in rapeseed on transcriptome level and screens out some environmentresponding candidate genes. 


\section{Investigation for a Multi-Silique Trait in Brassica}

\section{2 napus by Alternative Splicing Analysis}

3

4

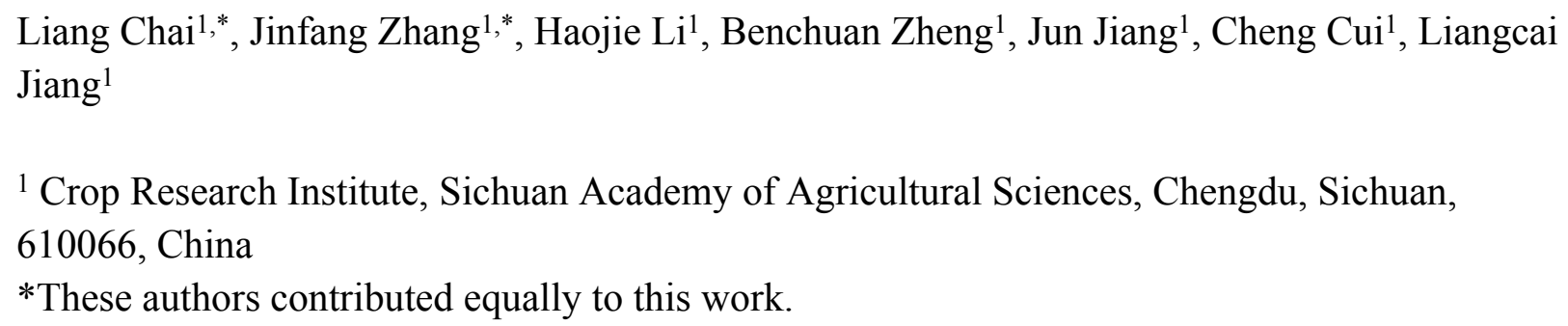

\section{Abstract}

Background. Flower and fruit development are vital stages of the angiosperm lifecycle. We previously investigated the multi-silique trait in the rapeseed (Brassica napus) line zws-ms on a genomic and transcriptomic level, leading to the identification of two genomic regions and several candidate genes associated with this trait. However, some events on transcriptome level, like alternative splicing, were poorly understood.

Methods. Plants from zws-ms and its near-isogenic line (NIL) zws-217 were both grown in Xindu with normal conditions and a colder area Ma'erkang. Buds from the two lines were sampled and RNA was isolated to perform the transcriptomic sequencing. The numbers and types of alternative splicing (AS) events from the two lines were counted and classified. Genes with AS events and expressed differentially between the two lines, as well as genes with AS events which occurred in only one line were emphasized. Their annotations were further studied. Results. From the plants in Xindu District, an average of 205,496 AS events, which could be sorted into 5 AS types, were identified. zws-ms and zws-217 shared highly similar ratios of each AS type: The alternative 5' and 3' splice site types were the most common, while the exon skipping type was observed least often. Eleven differentially expressed AS genes were identified, of which four were upregulated and seven were downregulated in zws-ms. Their annotations implied that five of these genes were directly associated with the multi-silique trait. While 
35

36

37

38

39

40

41

42

43

44

45

46

47

48

49

50

51

52

53

54

55

56

57

58

59

60

61

62

63

64

65

samples from colder area Ma'erkang generated generally reduced number of each type of AS events except the Intron Retention; but the number of differentially expressed AS genes increased significantly. Further analysis found that among the 11 differentially expressed AS genes from Xindu, three of them maintained the same expression models, while the other 8 genes did not show significant difference between the two lines in expression level. Additionally, the 205 line-specifically expressed AS genes were analyzed, of which 187 could be annotated, and two were considered to be important.

Discussion. This study provides new insights into the molecular mechanism of the agronomically important multi-silique trait in rapeseed on transcriptome level and screens out some environment-responding candidate genes.

\section{Introduction}

Rapeseed (Brassica napus L.), an allotetraploid with a complex genome (AACC, $2 \mathrm{n}=38$ ), is the second leading source of vegetable oil globally (Liu et al., 2015). The agronomic traits related to rapeseed yield include the pod (silique) number per plant, branch number, and seed weight (Liu et al., 2015; Zhang et al., 2006; Li et al., 2015). We previously reported that zws-ms, a multisilique rapeseed line (Chai et al., 2019), produces three independent pistils and 9 to 10 stamens on the same receptacle in a flower, which consequently leads to the formation of three independent siliques on a carpopodium rather than the single siliques typically observed. Moreover, this trait was found to be affected by the environment, with temperature considered to be the factor most likely to switch on/off the formation of multi-silique.

Temperate is a major environmental factor that regulates various aspects of plant morphology, physiology, and biochemistry, affecting germination, growth, development, and flowering (Ren et al., 2019). Fertility in crops such as rapeseed (Yu et al., 2015) and rice (Oryza sativa) (Yu et al., 2017) is affected by temperature. In winter rapeseed lines, although a period of vernalization under low temperature is necessary to initiate flowering, cold stress inhibits growth and development, disturbs metabolism, and causes wilting or even death. Notably, cold stress also induces alternative splicing (AS) in plants (Palusa et al., 2007; Iida, 2004).

4 AS is defined as the mechanism by which primary transcripts are processed into two or more mature isoforms, which enables a single gene to produce diverse protein products (Pan et al., 2008; Sablok et al., 2011). These proteins differ from each other not only in structure but also 
67 possibly in function, subcellular localization, and/or stability (Huang et al., 2019; Chauhan et 68 al., 2019). AS is common in plants; for example, in Arabidopsis thaliana, more than 60\% of 69 intron-containing genes undergo AS (Syed et al., 2012). Many environmental factors regulate AS 70 events in plants, including $\mathrm{CO}_{2}$ concentration (Huang et al., 2019), light (Godoy et al., 2019), 71 salt stress (Ding et al., 2014), and nutrient deficiencies (Nishida et al., 2017). AS not only 72 provides an important source of transcriptomic and proteomic diversity and plasticity for use in 73 natural selection (Labadorf et al., 2010), but it also plays specific roles in the response (Chauhan 74 et al., 2019) or adaptation to environmental stresses (Filichkin et al., 2015). Guo et al. (2019) 75 identified four splicing variants of two BnCYCD3-1-LIKE genes in B. napus and found evidence 76 that their AS may play an important role in the response to environmental stresses. Xia et al. 77 (2017) discovered that the AS with intron retention of EARLY MATURITY8 (EAM8) led to early 78 flowering in a barley (Hordeum vulgare) landrace; while in shepherd's purse (Capsella bursa79 pastoris), flowering time varies with changes in the splicing of a FLOWERING LOCUSC (FLC) 80 homolog (Slotte et al., 2009). In addition, the heterologous expression of a vacuolar membrane $81 \mathrm{Na}^{+} / \mathrm{H}^{+}$antiporter gene (SsNHXI) AS variant from seepweed (Suaeda salsa) enhances the salt 82 tolerance of Arabidopsis (Li et al., 2009).

As mentioned above, low temperatures switch off the multi-silique trait in zws-ms rapeseed. When zws-ms plants were planted in Xindu, Sichuan Province, China, the multi-silique trait was continuously stable for years; however, when they were grown in Ma'erkang, Sichuan Province, where the annual average temperature is consistently $7.6{ }^{\circ} \mathrm{C}$ lower, the multi-silique trait disappeared and all plants displayed normal siliques (Chai et al., 2019). We previously investigated the association of chromosomal regions with this trait, at the genomic and transcriptomic levels, selecting potential candidates from the differentially expressed genes (DEGs) between the multi- and single-silique plants. However, the involvement of posttranscriptional modifications and the mechanisms by which temperature regulates this multisilique trait remain unclear. AS is often responsive to cold stress in plants (Iida, 2004; Palusa et al., 2007) and is a mechanism by which plants perceive temperature fluctuations and modulate the activity of their transcription factors (Seo et al., 2013). In view of the above insights, we analyzed AS using transcriptome sequencing (RNA-seq) in this study. High-throughput RNAseq technology is a widely used, highly efficient, and economical strategy for transcriptomic 
98 the following qualities (Mortazavi et al., 2008; Ozsolak \& Milos, 2011; Marioni et al., 2008;

99 Tong et al., 2013; Sultan et al., 2008): (1) It can be used to detect and quantify the expression of 100 genes, including those expressed at low levels; (2) it can facilitate the annotation of genes and 101 lead to the discovery of novel genes or transcripts; (3) the results are highly reproducible 102 between both technical and biological replicates; and (4) it can detect AS events.

103 We performed transcriptome sequencing (RNA-seq) on the flower buds of zws-ms and its 104 near-isogenic line (NIL), zws-217, which produces normal single siliques. This facilitated the 105 identification of the AS events in both lines and the analysis of the differentially expressed AS 106 genes and those with line-specific AS events. Combining these data with Gene Ontology (GO) 107 and Kyoto Encyclopedia of Genes and Genomes (KEGG) annotations, we identified likely 108 candidate genes switching on or off the multi-silique trait by altering AS events or transcriptional 109 levels in varied environments.. To the best of our knowledge, this is the first time that the 110 regulation of flower/fruit morphology by AS has been investigated in rapeseed, and our results 111 provide insights into this field more generally.

114 Materials \& Methods

\section{Plant Materials and Growth Conditions}

116 The rapeseed line zws-ms and its NIL, zws-217 (Chai et al., 2019), were kept in the Crop 117 Research Institute, Sichuan Academy of Agricultural Sciences, China. Both zws-ms and zws-217 118 were homozygous for almost all genes, differing from each other only in the multi-silique trait of zws-ms (Figure 1). The NILs zws-217 and zws-ms were both grown in an experimental field in the Xindu District of Chengdu in the Sichuan Basin, China, under normal environmental conditions. Additionally, the both lines were also grown in Ma'erkang, a mountainous area in western Sichuan, with a much lower annual average temperature. The annual average temperature in Xindu and Ma'erkang is $16.2^{\circ} \mathrm{C}$ and $8.6^{\circ} \mathrm{C}$, respectively (Chai et al., 2019).

\section{Total RNA Extraction and Sequencing Library Construction}

Three zWs-ms plants (samples T01, T02, and T03) and three zws-217 plants (T04, T05, and T06) were selected for RNA isolation, as described previously (Chai et al., 2019). Flower buds 
129 extracted using an RNA Isolation Kit (Tiangen, Beijing, China). The quality and concentration of 130 the RNA were determined using a NanoDrop 2000 (Thermo Fisher Scientific, Waltham, MA, 131 USA), and the sequencing libraries were generated using an RNA Library Prep Kit for Illumina 132 (New England Biolabs, Ipswich, MA, USA).

133

134

135

136

137

138

139

140

141

142

143

144

145

146

147

148

149

150

151

152

153

154

155

156

157

158

159

\section{Sequencing and Expression Analysis}

The samples were sequenced on a HiSeq X Ten platform (Illumina, San Diego, CA, USA) and paired-end reads were generated. Low-quality reads and adaptor sequences were removed, and clean reads were used for the following analysis. TopHat2 (Kim et al., 2013) was used to map the clean reads onto the Brassica napus reference genome (Chalhoub et al., 2014) with default parameters “--read-mismatches 2 --read-edit-dist 2 --library-type fr- --max-intron-length 5000000". The number of fragments per kilobase of transcripts per million fragments mapped (FPKM) was calculated to represent the gene expression level, and the DESeq R package (Anders \& Huber, 2010) was used to analyze the differential expression. The P-value was adjusted using Benjamini and Hochberg's approach to control the false discovery rate (FDR). The relative expression levels of each transcript calculated using DESeq were used to define the DEGs, which were defined as having a fold change $>4$ and an FDR $<0.01$. Pearson's correlation coefficients were determined for the three biological replicates of each line to determine the reliability of the DEGs. Moreover, real-time quantitative PCR (qPCR) was performed to validate the transcriptome sequencing. Since the validation for transcriptome sequencing data from plants in Xindu had been confirmed previously (Chai et al., 2019), we only validated the data from plants in colder Ma'erkang herein. Amplification reactions were performed on iQ $^{\mathrm{TM}} 5$ Real-Time PCR System (Biorad) as follows: an initial denaturation step at $95^{\circ} \mathrm{C}$ for $3 \mathrm{~min}, 39$ cycles at 95 ${ }^{\circ} \mathrm{C}$ for $10 \mathrm{~s}, 60{ }^{\circ} \mathrm{C}$ for $30 \mathrm{~s}$, and $72{ }^{\circ} \mathrm{C}$ for $30 \mathrm{~s}$. After each run, a melt curve was acquired to check for amplification specificity by heating the samples from $60^{\circ} \mathrm{C}$ to $95{ }^{\circ} \mathrm{C}$. Three biological replicates were applied.

\section{AS Event Analysis}

The cleaned sequence data were aligned to the reference genome using TopHat2 (Kim et al., 2013) with default settings mentioned above. The resultant gapped alignment data in a binary 
160 alignment format were then used as an input for Cufflinks and Cuffcompare, which were run 161 using the default settings to assemble the transcripts and identify splicing junctions from the 162 alignment data. For the AS detection and annotation, the AS events were annotated with 163 ASprofile (Florea et al., 2013), which uses Cufflinks and Cuffcompare outputs as input data. 164 Default parameters of the software were used.

165

166

\section{Annotation of Genes}

167

168

169

170

171

172

173

174

175

176

177

178

179

180

181

182

183

184

185

186

187

188

189

190

Gene function was annotated based on the following databases: $\mathrm{Nr}$ (NCBI nonredundant protein sequences), Nt (NCBI nonredundant nucleotide sequences), Pfam (Protein family), KOG/COG (Clusters of Orthologous Groups of proteins), Swiss-Prot (a manually annotated and reviewed protein sequence database), KO (KEGG Ortholog database), and GO (Gene Ontology).

The GO enrichment analysis of the DEGs was performed using the GOseq R packages based on a Wallenius noncentral hypergeometric distribution (Young et al., 2010), which can adjust for gene length bias in the DEGs.

The KEGG database (Kanehisa et al., 2007) is a resource used to explore the high-level functions and utilities of the biological system, such as the cell, organism, and ecosystem, from molecular-level information, especially using large-scale molecular datasets generated from genome sequencing and other high-throughput experimental technologies (http://www.genome.jp/kegg/). KOBAS (Mao et al., 2005) software was used to test the statistical enrichment of the DEGs in the various KEGG pathways. Default parameters were used.

\section{Results}

\section{Transcriptome Sequencing}

Flower buds from three plants of both the multi-silique line zws-ms and the single-silique NIL zWs-217 (Figure 1) were sampled for RNA extraction. The sequencing saturation and cluster analysis of the samples were determined to ensure the validity of the data. In total, $65.6 \mathrm{~Gb}$ of clean data were generated, with an average Q30 value of 90.54\%. Each sample generated about 36.65 M clean reads with an average GC content of 47.23\% (Table S1). The average proportion of total reads mapped to the reference genome for each sample was $73.72 \%$ (Table S2). 
191 Validation of this transcriptome sequencing data was previously confirmed by qPCR (Chai et al., 192 2019). Similarly, samples from colder area Ma'erkang also generated abundant data, which was 193 validated by comparing the relative transcript levels of eight DEGs in zws-ms and zws-217 by 194 qPCR. The qPCR analysis (Figure S1) showed that all genes had similar trends in expression as 195 those observed by transcriptome sequencing (described below). Each sample generated about $19622.92 \mathrm{M}$ clean reads with an average GC content of 46.27\%, and Q30 value of 92.95\% (Table 197 S3); average proportion of total reads mapped to the reference genome for each sample was 198 88.87\% (Table S4).

199

\section{AS Event Identification and Analysis}

201 According to description by Reddy (2007), alternative splicing events were sorted into 5 classes: 202 Alternative 3' splice site, Alternative 5' splice site, Exon Skipping, Intron Retention and 203 Mutually Exclusive Exons. The six samples grown in Xindu under normal conditions displayed 204 an average of 205,496 AS events (Table 1; Table S5). The proportions of each AS type were 205 analyzed in both zws-ms and zws-217. The two lines shared highly similar ratios of each AS 206 type, with the alternative 5' splice site and alternative 3' splice site types being the most 207 commonly observed, at $43.48 \%$ and $42.77 \%$ of AS events for both lines, respectively. The

208

209

210

211

212

213

214

215

216

\section{Annotation of the Alternatively Spliced Genes}

218 To study the biological functions of the genes with AS events, GO and KEGG pathway 219 enrichment analyses were performed. The GO annotations for AS genes from plants in Xindu 220 221

mutually exclusive exons type was the next most common (6.61\%), followed by the Intron Retention type (5.92\%), and the least common types was Exon Skipping, which represented just $1.22 \%$ of the AS events (Figure 2a; Table 1).

As to the plants grown in colder area Ma'erkang, the two lines also shared highly similar ratios of each AS type: the alternative 5' splice site and alternative 3' splice site types represented the greatest proportion, at $42.13 \%$ and $41.29 \%$, respectively; while the exon skipping accounted least proportion 1.51\% (Figure 2b; Table 1). The number of each type of AS events were significantly reduced in colder area Ma’erkang, except the Intron Retention. included 17 terms involved in biological processes (BP; Figure 3a), 17 terms associated with cellular components (CC), and 20 terms involved in molecular functions (MF). The most highly

Peer] reviewing PDF | (2019:12:44287:4:0:NEW 6 Sep 2020) 
222 enriched BP terms observed in the alternatively spliced genes included "cellular process", 223 "single-organism process" and "metabolic process". The most common CC categories were 224 "cell", "cell part" and "organelle". In the MF category, the most enriched terms were "binding", 225 "catalytic activity" and "nucleic acid binding transcription factor activity". Plants grown in 226 Ma'erkang showed highly similar GO data to that in Xindu: 22 terms involved in BP (Figure 3b), 22715 terms associated with CC, and 15 terms involved in MF. Moreover, data from Ma'erkang and 228 Xindu showed the same top-3 most enriched terms in each category.

229 These KEGG pathways were classified into five major groups: metabolism, genetic 230 information processing, cellular processes, environmental information processing, and 231 organismal systems. Of these, the subgroups "biosynthesis of amino acids", "carbon 232 metabolism", "ribosome", and "RNA transport" contained the highest number of annotated 233 genes (Figure 4a). Data from Ma'erkang showed similar subgroups containing the most of AS 234 genes: "ribosome", "carbon metabolism", "biosynthesis of amino acids" and "plant hormones 235 signal transduction" contained the highest number of annotated genes (Figure 4b).

236

\section{DEGs with AS and Their Arabidopsis Orthologs}

238 DESeq software was used to identify the different expression levels of the AS genes in zws-ms 239 and zws-217. From Xindu, eleven differentially expressed AS genes were identified, of which 240 four were upregulated and seven were downregulated in zws-ms (Table 2).

241 The Arabidopsis orthologs of these differentially expressed AS genes were identified using 242 The Arabidopsis Information Resource (TAIR; https:/www.arabidopsis.org; Table 3). The 243 following orthologs were identified: (1) AT5G15470, the ortholog of BnaA02g02630D, encodes 244 galacturonosyltransferase 14 (GAUT14); (2) AT3G15420 encodes the transcription factor TFIIIC 245 (tau55-related protein); (3) AT1G14800 encodes a nucleic acid-binding, OB-fold-like protein; (4) 246 AT2G04900 encodes an unknown protein; (5) AT3G10070 encodes one of two Arabidopsis 247 proteins with similarity to the TBP-associated factor, TAF12; (6) AT1G15060 encodes an 248 alpha/beta hydrolase family protein; (7) AT3G54620 encodes a bZIP transcription factor-like 249 protein; (8) AT5G16210 encodes a HEAT repeat-containing protein; (9) AT3G59000 encodes an 250 F-box/RNI-like superfamily protein; (10) AT4G16900, the ortholog of BnaC07g33980D, 251 encodes a member of the disease resistance protein (TIR-NBS-LRR class) family; and (11) 252 AT1G10760 encodes an $\alpha$-glucan, water dikinase (GWD) required for starch degradation. 
253 When grown in Ma'erkang, the two lines generated increased number of differentially 254 expressed AS genes significantly to 130 (Table S6), including 52 unregulated and 78 down 255 regulated AS genes. Four AS genes were annotated to "response to cold (GO:0009409)": 256 BnaAnng17190D, BnaC01g27600D, BnaC08g39130D and BnaC09g53990D; three AS genes, 257 BnaA07g19340D, BnaC01g27600D, BnaC08g39130D, were annotated to "response to heat 258 (GO:0009408)"; three were related to "response to freezing (GO:0050826)", including 259 BnaA05g28590D, BnaC06g15710D and BnaC08g36010D; BnaCnng24040D was found relevant 260 to "temperature stimulus (GO:0009266)". Moreover, BnaC08g36010D, BnaC08g39130D and 261 BnaC08g39360D were annotated to "regulation of flower development (GO:0009909)", "plant 262 ovule development (GO:0048481)" and "fruit development (GO:0010154)", respectively (Table 263 S6). Compared with the 11 differentially expressed AS genes from normal conditions in Xindu, 264 three of them (BnaA07g04500D, BnaAnng30260D and BnaC06g16950D) maintained the same 265 expression models. In other words, these three genes were upregulated under both normal and 266 colder conditions. While the other 8 genes (BnaA02g02630D, BnaA02g03080D, 267 BnaA04g16220D, BnaA09g45000D, BnaA09g45260D, BnaC02g06440D, BnaC07g33980D and 268 BnaC08g49610D) did not show significant difference between zms-ms and zws-217 in 269 expression level in Ma'erkang.

270

\section{Genes with Line-specific AS Events}

272 Genes with line-specific AS events, defined as those genes with a particular AS event(s) that 273 occurred only in zws-ms or in zws-217, were also identified and analyzed. Unlike the above274 mentioned general classifications, we sorted AS events into 12 finer subclasses, in order to 275 identify them more specifically: transcription start site (TSS) and transcription terminal site 276 (TTS) equaled to original Alternative 5' first exon and Alternative 3' last exon, respectively; 277 Mutually exclusive exons was subdivided into Alternative exon ends (AE) and Approximate AE 278 (XAE); the original Intron retention was subdivided into single Intron retention (IR), 279 Approximate IR (XIR), Multi-IR (MIR) and Approximate MIR (XMIR); the Cassette exon was 280 then was subdivided into single Skipped exon (SKIP), Approximate SKIP (XSKIP), Multi-exon 281 SKIP (MSKIP) and Approximate MSKIP (XMSKIP). In total, 205 line-specifically expressed 282 AS genes were detected, of which 187 could be annotated (Table S7). Eight genes related to 283 "ovule development", "flower morphogenesis" and other similar processes were highlighted and 
284 considered important for further study in the coming future (Table 4, Table S7): (1) An IR event 285 (32749874- 32749905 bp on chromosome C06) of BnaC06g32640D occurred line-specifically in 286 zws-217 in Xindu, while this event disappeared in both lines in Ma'erkang. Furthermore, a TSS 287 event (32750089-32750270 bp on chromosome C06) only happening in multi-silique zws-ms in 288 Xindu, appeared in both lines when planted in Ma'erkang. This gene was annotated as 289 "vegetative to reproductive phase transition of meristem (GO:0010228)" and "Biological 290 Process: ovule development (GO:0048481)"; (2) BnaC07g00780D was associated with 291 "reproductive structure development (GO:0048608)". In Xindu, two specific AE events 292 (1070954- 1071329 bp and 1070976- 1071329 bp on chromosome C07, respectively) of it were 293 observed in zws-217, but they both disappeared in two lines when planted in Ma'erkang; (3) 294 BnaC04g31460D and (4) BnaC05g34570D were related to "regulation of flower development 295 (GO:0009909)". An SKIP event (33329566- 33329615 bp on chromosome C04) for 296 BnaC04g31460D and an IR event (33879446-33879532 on chromosome C05) for 297 BnaC05g34570D were identified only in zws-217 from Xindu; Similarly, two AE events 298 (27558813- 27558932 bp and 27558838- 27558932 bp on chromosome C04) for (5) 299 BnaC04g26180D, two IR events (31448257-31448346 bp and 31448264- 31448353 bp on 300 chromosome C07) for (6) BnaC07g25280D and one IR event (19819830-19820358 bp on 301 chromosome C07) for (7) BnaC03g32190D were all found line-specificaly in zws-217 from 302 Xindu, and none of them were identified in either liens from Ma'erkang. BnaC04g26180D was 303 annotated with "development (GO:0048481)"; BnaC07g25280D was annotated as "flower 304 morphogenesis; organ morphogenesis (GO:0009887)" and "vegetative to reproductive phase 305 transition of meristem (GO:0010228)"; BnaC03g32190D was annotated as "double fertilization 306 forming a zygote and endosperm (GO:0009567)"; (8) BnaCnng68400D was associated with 307 "carpel development (GO:0048440)." An IR and two AE events of this gene were detected 308 specifically in multi-silique line under normal conditions, while in colder area, they were not 309 identified in neither zws-ms nor zws-217.

310

311

\section{Discussion}

313 As an important post-transcriptional metabolic event, AS is involved in many plant growth and 314 developmental processes, such as flowering induction (Eckardt, 2002; Slotte et al., 2009) and the 
315 responses to environmental fluctuations and pathogen attacks (Barbazuk et al., 2008). To the best 316 of our knowledge, AS events have seldom been reported to regulate the development of 317 flower/fruit morphology in higher plants. This study is the first to analyze the role of AS events 318 in rapeseed flower/fruit development as a whole, let alone those related to the multi-silique trait.

319 We previously described the morphology and inheritance of the multi-silique trait in $B$. 320 napus (Jiang et al., 1998), investigating the associated regions of chromosomes at the genomic 321 level and transcriptomically exploring the DEGs in multi-silique and single-silique plants (Chai 322 et al., 2019). The multi-silique trait was found to be controlled by three recessive alleles and was 323 significantly affected by environment; however, the mechanisms by which environmental factors 324 affect this trait remained unknown, even if we knew that temperature could switch on/off the 325 multi-silique trait (Chai et al., 2019). As mentioned above, AS is a pathway by which the 326 environment could regulate plant physiology, therefore in this study, we analyzed AS events in

327 328 329 330

331

332 333 order to investigate the mechanism by which plants perceive temperature fluctuations.

In this study, we sampled the buds of three individual plants from zws-ms and zws-217 lines in both Xindu and colder area Ma'erkang, and then subjected them to RNA-seq. All of the four groups generated sufficient data, which was validated by qPCR in earlier and present study successively. The samples in Ma'erkang group generated less data, mainly due to the less sequencing depth; nevertheless, this still provided enough data of high quality and assured the accuracy of the subsequent analysis.

We identified all of the genes with AS events in the zws-ms and zws-217 plants. In Xindu group, 11 AS genes were significantly differentially expressed between the multi-silique zws-ms line and its NIL, zws-217, which produces normal siliques. We analyzed their annotations and orthologs in Arabidopsis. One such ortholog, AT5G15470 (also known as Galacturonosyltransferase 14, GAUT14), is involved in cell wall pectin biosynthesis (Caffall et al., 2009), and the gaut13 gaut14 double mutant was previously shown to be defective in pollen tube growth (Wang et al., 2013). AT3G15420 (the ortholog of BnaA02g03080D) and $A T 3 G 10070$ (the ortholog of BnaA09g45000D) encode subunits of the transcription factor complexes TFIIIC and TAF12, respectively. The former does not appear to be substantially involved in plant development; however, some members of the TAF family are involved in the regulation of morphology. The transgenic expression of TAF10 from clustered yellowtops (Flaveria trinervia) in Arabidopsis limited the development of the indeterminate inflorescence 
346 and resulted in the production of deformed leaves (Furumoto et al., 2005). By contrast, the taf 347 mutant in Arabidopsis has abnormal phyllotaxis and lacks proper vegetative meristem activity 348 (Tamada et al., 2007), indicating the important roles played by the TAFs in plant morphological 349 development. Another DEG AS gene, BnaA04g16220D, is not annotated, and its Arabidopsis 350 ortholog AT1G14800 is simply listed as an uncategorized nucleic acid-binding, OB-fold-like 351 protein. The AS gene orthologs AT2G04900 and AT1G15060 encode an unknown protein and an 352 uncategorized alpha/beta hydrolase family protein, respectively, so their roles in the regulation of the multi-silique trait are also currently unclear.

Another ortholog for differentially expressed AS gene, AT3G54620, is reported to encode a 355 bZIP transcription factor-like protein. Members of this protein family are typically reported to regulate plant tolerance of environmental stresses. The transgenic expression of the maize (Zea 357 mays) gene ZmbZIP72 in Arabidopsis enhanced its drought and salt tolerance (Ying et al., 2012), while BnbZIP3, a ramie (Boehmeria nivea) bZIP transcription factor, also increased the drought, salinity, and heavy metal tolerances of transgenic Arabidopsis (Huang et al., 2016). These genes 360 are also involved in the regulation of other processes; for example, the repression of a bZIP 361 transcription factor gene OSABI5 expression in rice resulted in low fertility (Zou et al., 2008), while the transgenic expression of tomato (Solanum lycopersicum) SlbZIP2 in tobacco (Nicotiana benthamiana) increased leaf thickness (Seong et al., 2016). To date, however, there 364 are no reports of $b Z I P$ genes playing a significant role in flower/fruit morphology.

Other AS gene orthologs included AT5G16210, encoding a member of the HEAT repeat366 containing protein family, which are considered to be involved in intracellular transport 367 (Hernández-Torres et al., 2014; Oeffinger et al., 2004). Although BnaC06g16950D is not 368 annotated, its ortholog, AT3G59000, was identified as encoding an F-box/RNI-like superfamily protein in Arabidopsis, which typically function in the plant hormone signaling pathways (Gao et 370 al., 2009). Similarly, the ortholog AT4G16900 encodes a TIR-NBS-LRR class protein, which are known to be involved in disease resistance (Xun et al., 2019) and hormonal responses (Sarazin et 372 al., 2015). Moreover, Hewezi et al. (2006) unexpectedly found that these proteins are associated 373 with developmental abnormalities; transgenic sunflowers (Helianthus annuus) expressing the 374 antisense sequence complementing PLFOR48, which encodes a TIR-NBS-LRR-type protein, 375 showed stunted growth and a reduction in apical dominance; whereas the pods of transgenic 376 tobacco ( $N$. tabacum) lacking PLFOR48 expression were smaller and showed severe 
377 deformations. This indicates that TIR-NBS-LRR-type proteins can regulate the morphology of 378 plants, including fruit morphology, to some extent. Finally, AT1G10760, the ortholog of AS gene 379 BnaC08g49610D, which encodes a GWD protein required for starch degradation, is involved in 380 carbohydrate metabolism (Nadolska-Orczyk et al., 2017). This gene was also reported to regulate 381 seed size; Pirone et al. (2017) found that the length and width of the mature seeds were reduced 382 in the gwdl Arabidopsis mutant, while their density was increased.

383

384 385 386 387 388 389 390 391

392

393 394 395 396 397 398 399 400 401 402 403 404 405 406 407

To summarize, AT5G15470, AT3G10070, AT3G54620, AT4G16900, and AT1G10760 are all known to be involved in plant development; therefore, their corresponding rapeseed orthologs, BnaA02g02630D, BnaA09g45000D, BnaAnng30260D, BnaC07g33980D, and BnaC08g49610D, the expression levels of which differed significantly between zws-ms and zws-217, are considered to be potential candidate genes regulating the multi-silique trait.

After that, we continued to investigate data from Ma'erkang, where it is colder and the multi-silique trait in zws-ms line disappeared. Due to the importance of the above-mention 11 differentially expressed AS genes, we first paid attention to them and found that three of them had the same expression models as in Xindu. In other words, they were independent of temperature; in addition, combined with their annotations, they were excluded from the potential candidate genes responding to environmental factors. On the other hand, other 8 AS genes stopped being differentially expressed between zms-ms and zws-217 in Ma'erkang. That is to say, the expression level of these 8 AS genes (BnaA02g02630D, BnaA02g03080D, BnaA04g16220D, BnaA09g45000D, BnaA09g45260D, BnaC02g06440D, BnaC07g33980D and BnaC08g49610D) were environment-specifc. Besides, BnaC08g36010D, BnaC08g39130D and BnaC08g39360D, which were annotated to flower/ovule/fruit-related terms, were differentially expressed between zws-ms and zws-217 specifically in Ma'erkang. Moreover, we also found 9 temperature-responding AS genes differentially expressed in colder area: BnaAnng17190D, BnaC01g27600D, BnaC08g39130D, BnaC09g53990D, BnaA07g19340D, BnaA05g28590D, BnaC06g15710D, BnaC08g36010D and BnaCnng24040D. The lower temperature motivated more responding genes and this also explained the reason for increased number of AS genes clustered in each KEGG pathway.

We also explored the line-specific AS genes, which were similarly expressed between zwsms and zws-217, but contained stable and particular AS event(s) that differed between these two lines. These genes are likely to qualitatively regulate the multi-silique trait. In this case, we could 
408 obtain better results by fine-classify the AS types into 12 subclasses, rather than 5 classes 409 mentioned above. Because fine classifications could better identify differences between AS types 410 more precisely and subtly. Thus, we found 205 genes of this type, of which 187 could be 411 annotated. Due to the rarity of the multi-silique trait, we did not obtain much useful information 412 from the KEGG pathway analysis. This meant that we were unable to relate this metabolic 413 pathway information to the multi-silique trait directly; however, the GO analysis provided more 414 potential clues. Among these, eight genes were considered to be associated with 415 flower/carpel/ovule development. BnaC06g32640D is annotated as being involved in the 416 regulation of the vegetative-to-reproductive phase transition in the meristem (GO:0010228) and 417 in ovule development (GO:0048481). The IR of it in zws-217 seemed to block the multi-silique, 418 while the TSS occurred in zWs-ms as if it was positively related to mulsi-silique trait. Its 419 Arabidopsis ortholog, AT1G71692, is annotated as AGAMOUS-LIKE12 (AGL12). Peng et al. 420 (2015) isolated the BnFUL gene in rapeseed, which is homologous to AGL8 in Arabidopsis. 421 Although BnFUL was hypothesized to be involved in enhancing pod-shattering resistance, when 422 introduced into Arabidopsis, two of the five transgenic plants expressing BnFUL unexpectedly 423 had a multi-silique phenotype. However, the mechanisms by which BnFUL generates this multi424 silique phenotype remain elusive thus far, making the AGL12 gene identified in this study a 425 potentially important candidate gene. The ortholog gene of BnaCnng68400D, AT5G15020, 426 encodes an SIN3-LIKE 2 protein (SNL2) known to be important for seed germination or 427 dormancy (Wang et al., 2016; Wang et al., 2013). The zWs-ms line-specific AS events of it were 428 detected in Xindu, where the two NILs were distinct from each other in the multi-silique trait, 429 while in Ma'erkang, where the distinguishing trait was switched off, these AS event disappeared 430 in zws-ms. This showed a positive correlation to the trait.

431 The other six selected line-specific AS genes showed particular AS events in zws-217 in 432 Xindu, implying potential inhibition of the multi-silique morphology. However, these AS events 433 did not occur in zws-ms or zws-217 in Ma'erkang, representing unknown complexity of the 434 mechanisms, which were strongly influenced by environment. Fortunately, their orthologs in 435 Arabidopsis provided many useful clues. AT2G20180 and AT4G00050, orthologs of 436 BnaC07g00780D and BnaC03g32190D respectively, both encode phytochrome interacting 437 factors (PIFs). Several transcription factors (AP1, SVP, LFY, AG, and SEP3) involved in the 438 regulation of flowering are known to bind to the PIFs, suggesting a direct link with the reported 
439 flowering phenotype of the pif mutants (Leivar \& Monte, 2014). AT5G17270 encodes a 440 prenylyltransferase superfamily protein; however, to the best of our knowledge, there have been 441 no reports about its development-related functions. The Arabidopsis ortholog of 442 BnaC05g34570D is $A T 3 G 18600$, which encodes a P-loop-containing nucleoside triphosphate 443 hydrolase. While few studies have reported the functions of these proteins, Liu et al. (2016) 444 reported that, in sesame (Sesamum indicum), one gene encoding a P-loop-containing nucleoside 445 triphosphate hydrolase showed a reduced expression level in sterile buds, indicating that they 446 may play a role in specifying/determining tapetal fate and development. Another line-specific 447 AS ortholog, AT3G54660, encodes a glutathione reductase (GR), which was found to increase 448 the fineness (mass per unit length) and bundle strength of cotton (Gossypium hirsutum) fiber 449 when transgenically expressed (Tuttle et al., 2015). Since cotton fibers are single cells initiating 450 from the epidermis of the outer integument of the ovules (Ruan et al., 2004), it can be inferred 451 that GR regulates ovule development to some extent. AT3G28730, also known as structure452 specific recognition protein SSRP1, was also found to regulate floral development, as the ssrp 1-2 453 mutant Arabidopsis produced small and deformed petals with shorter stamens (Lolas et al., 454 2010).

455 To date, there is some evidence to show that the line-specific AS orthologs AT2G20180, $456 A T 4 G 00050$ and $A T 3 G 54660$ are related to the regulation of flower/fruit morphology, with clear 457 roles reported for AT1G71692 and AT3G28730. Consequently, their orthologs in rapeseed, 458 BnaC06g32640D and BnaC07g25280D, respectively, are considered to be important candidate 459 genes regulating the multi-silique trait by conferring or removing some specific line-specific AS 460 461 events in varied environments. In addition, BnaCnng68400D, of which AS events represented a positive correlation to morphology with or without multi-siliques, was also noteworthy.

Some of the genes/loci controlling silique development in Brassica plants have previously been reported. In addition to those regulating traits such as the seed weight and silique length 464 (Liu et al., 2015) and the number of seeds per silique in B. napus (Li et al., 2015), some genes 465 related to silique morphology have been cloned and functionally analyzed. Xiao et al. (2013) 466 fine-mapped a multi-locular silique gene, Bjln1, to a $208-\mathrm{kb}$ region on chromosome A7 in 467 Brassica juncea and then revealed that it was the mutations in the CDS and promoter of 468 BjuA07.CLV1 gene (equivalent to Bjln1) to cause the multi-locular trait (Xiao et al., 2018). Both 469 Fan et al. (2014) and Yadava et al. (2014) reported that a mutation in BrCLV3, a homologue of 
470 CLAVATA3 in Arabidopsis, caused the production of multi-locular siliques in B. rapa. However, 471 the multi-silique (or multi-pistil) phenotype of zws-ms is different from the above-motioned 472 multi-locular trait; zws-ms produces three pods on each carpopodium, rather than multiple loculi 473 per pod.

474 Few studies have investigated this multi-silique trait in rapeseed; however, there have been 475 similar reports of multi-pistil traits in other crops, particularly in wheat (Triticum aestivum): 476 Duan et al. (2015) discovered a male-sterile wheat mutant, dms, with a dwarf status and multi477 pistils, a pleiotropic phenotype found to be controlled by a single recessive gene, which was not 478 identified. Guo et al. (2019) reported another multi-ovary trait in the wheat line DUOII, which 479 was controlled by a dominant gene, and used a proteomics approach to propose some candidate 480 proteins. Yang et al. (2017) mapped a gene promoting the formation of three pistils (Pis 1) to 481 chromosome 2D and identified some candidate genes according to their annotations, while Zhu 482 et al. (2019) discovered a wheat multi-pistil mutant, 12TP, which was found to contain a 483 semidominant mutation located on chromosome arm 2DL. Although several studies have 484 explored the multi-pistil trait in wheat, no one has identified any of the specific genes responsible 485 yet.

To sum up, the seven candidate genes mentioned above, including the five differentially 487 expressed AS genes of interest (BnaA02g02630D, BnaA09g45000D, BnaAnng30260D, 488 BnaC07g33980D, and BnaC08g49610D) and the two genes with line-specific AS events (BnaC06g32640D and BnaC07g25280D), are therefore hypothesized to regulate the multisilique trait in rapeseed zws-ms, based on their AS expression levels or line-specific AS events altered by environment. These findings lay a foundation for further functional analyses in future.

\section{Conclusions}

495 The utilization of heterosis is a way to increase the yield or improve the quality of crops. 496 Exploring new germplasm resources and genes, as well as clarifying their inheritance, is the 497 foundation of obtaining of excellent hybrid. This study provides a novel inspection into the 498 multi-silique trait in rapeseed from the transcriptional perspective by AS responding to 499 environment, deepening the understanding of its molecular mechanism. Further function 500 verifications are now undergoing. 
502

503

504

505

506

507

508

509

510

511

512

513

514

515

516

517

518

519

520

521

522

523

524

525

526

527

528

529

530

531

532

533

534

535

536

537

538

539

540

541

542

543

544

545

546

\title{
Acknowledgements
}

\author{
$\mathrm{N} / \mathrm{A}$
}

\section{References}

Anders S, and Huber W. 2010. Differential expression analysis for sequence count data. Genome Biology 11:R106. DOI 10.1186/gb-2010-11-10-r106

Barbazuk WB, Fu Y, and McGinnis KM. 2008. Genome-wide analyses of alternative splicing in plants: Opportunities and challenges. Genome Research 18:1381-1392. DOI 10.1101/gr.053678.106

Caffall KH, Pattathil S, Phillips SE, Hahn MG and Mohnen D. 2009. Arabidopsis thaliana T-DNA Mutants Implicate GAUT Genes in the Biosynthesis of Pectin and Xylan in Cell Walls and Seed Testa. Molecular Plant 2:1000-1014. DOI 10.1093/mp/ssp062

Chai L, Zhang J, Lu K, Li H, Wu L, Wan H, Zheng B, Cui C, Jiang J and Jiang L. 2019. Identification of genomic regions associated with multi-silique trait in Brassica napus. BMC Genomics 20:304. DOI 10.1186/s12864-019-5675-4

Chalhoub B, Denoeud F, Liu S, Parkin IA, Tang H, Wang X, Chiquet J, Belcram H, Tong C, Samans B, Correa M, Da SC, Just J, Falentin C, Koh CS, Le Clainche I, Bernard M, Bento P, Noel B, Labadie K, Alberti A, Charles M, Arnaud D, Guo H, Daviaud C, Alamery S, Jabbari K, Zhao M, Edger PP, Chelaifa H, Tack D, Lassalle G, Mestiri I, Schnel N, Le Paslier MC, Fan G, Renault V, Bayer PE, Golicz AA, Manoli S, Lee TH, Thi VH, Chalabi S, Hu Q, Fan C, Tollenaere R, Lu Y, Battail C, Shen J, Sidebottom CH, Wang X, Canaguier A, Chauveau A, Berard A, Deniot G, Guan M, Liu Z, Sun F, Lim YP, Lyons E, Town CD, Bancroft I, Wang X, Meng J, Ma J, Pires JC, King GJ, Brunel D, Delourme R, Renard M, Aury JM, Adams KL, Batley J, Snowdon RJ, Tost J, Edwards D, Zhou Y, Hua W, Sharpe AG, Paterson AH, Guan C and Wincker P. 2014. Early allopolyploid evolution in the post-Neolithic Brassica napus oilseed genome. Science 345:950-953. DOI 10.1126/science.1253435

Chauhan K, Kalam H, Dutt R and Kumar D. 2019. RNA Splicing: A New Paradigm in Host-Pathogen Interactions. Journal of Molecular Biology 431:1565-1575. DOI 10.1016/j.jmb.2019.03.001

Ding F, Cui P, Wang Z, Zhang S, Ali S and Xiong L. 2014. Genome-wide analysis of alternative splicing of pre-mRNA under salt stress in Arabidopsis. BMC Genomics 15:431. DOI 10.1186/1471-2164-15-431

Duan Z, Shen C, Li Q, Lü G, Ni Y, Yu D and Niu J. 2015. Identification of a novel male sterile wheat mutant $d m s$ conferring dwarf status and multi-pistils. Journal of Integrative Agriculture 14:1706-1714. DOI 10.1016/S2095-3119(14)60936-9

Eckardt NA. 2002. Alternative splicing and the control of flowering time. Plant Cell 14:743-747. DOI $10.1105 /$ tpc. 000000

Fan C, Wu Y, Yang Q, Yang Y, Meng Q, Zhang K, Li J, Wang J and Zhou Y. 2014. A Novel SingleNucleotide Mutation in a CLAVATA3 Gene Homolog Controls a Multilocular Silique Trait in Brassica rapa L. Molecular Plant 7:1788-1792. DOI 10.1093/mp/ssu090

Filichkin S, Priest HD, Megraw M and Mockler TC. 2015. Alternative splicing in plants: directing traffic at the crossroads of adaptation and environmental stress. Current Opinion in Plant Biology 24:125-135. DOI 10.1016/j.pbi.2015.02.008

Florea L, Song L, Salzberg SL. 2013. Thousands of exon skipping events differentiate among splicing patterns in sixteen human tissues. F1000Research 2:188. DOI 10.12688/f1000research.2-188.v2

Furumoto T, Tamada Y, Izumida A, Nakatani H, Hata S and Izui K. 2005. Abundant Expression in Vascular Tissue of Plant TAF10, an Orthologous Gene for TATA Box-binding Protein-associated Factor

Peer) reviewing PDF | (2019:12:44287:4:0:NEW 6 Sep 2020) 
547

548

549

550

551

552

553

554

555

556

557

558

559

560

561

562

563

564

565

566

567

568

569

570

571

572

573

574

575

576

577

578

579

580

581

582

583

584

585

586

587

588

589

590

591

10, in Flaveria trinervia and Abnormal Morphology of Arabidopsis thaliana Transformants on its Overexpression. Plant and Cell Physiology 46:108-117. DOI 10.1093/pcp/pci006

Gao Y, Zhao Y, Li T, Liu Y, Ren C and Wang M. 2009. Molecular cloning and expression analysis of an Fbox protein gene responsive to plant hormones in Brassica napus. Molecular Biology Reports 37:1037. DOI 10.1007/s11033-009-9822-x

Godoy HM, Kubaczka MG, Brzyzek G, Servi L, Krzyszton M, Simpson C, Brown J, Swiezewski S, Petrillo E, and Kornblihtt AR. 2019. Light Regulates Plant Alternative Splicing through the Control of Transcriptional Elongation. Molecular Cell 73:1066-1074. DOI 10.1016/j.molcel.2018.12.005

Guo J, Zhang G, Song Y, Li Z, Ma S, Niu N and Wang J. 2019. Comparative proteomic analysis of multiovary wheat under heterogeneous cytoplasm suppression. BMC Plant Biology 19:175. DOI 10.1186/s12870-019-1778-y

Guo Y, Li J, Fang Y, Wan Y, Tang J, Wei T, Jiang X, Wang R and Wang M. 2019. An event of alternative splicing affects the expression of two BnCYCD3-1-like genes in Brassica napus. Gene 694:3341. DOI 10.1016/j.gene.2018.12.085

Hernández-Torres J, Jaimes-Becerra AJ and Chomilier J. 2014. Arabidopsis thaliana Tic110, involved in chloroplast protein translocation, contains at least fourteen highly divergent heat-like repeated motifs. Biologia 69:139-151. DOI 10.2478/s11756-013-0310-3

Hewezi T, Mouzeyar S, Thion L, Rickauer M, Alibert G, Nicolas P and Kallerhoff J. 2006. Antisense Expression of a NBS-LRR Sequence in Sunflower (Helianthus annuus L.) and Tobacco (Nicotiana tabacum L.): Evidence for a Dual Role in Plant Development and Fungal Resistance. Transgenic Research 15:165-180. DOI 10.1007/s11248-005-3518-3

Huang C, Zhou J, Jie Y, Xing H, Zhong Y, She W, Wei G, Yu W and Ma Y. 2016. A ramie (Boehmeria nivea) bZIP transcription factor BnbZIP3 positively regulates drought, salinity and heavy metal tolerance. Molecular Breeding 36:120. DOI 10.1007/s11032-016-0470-2

Huang W, Chen X, Guan Q, Zhong Z, Ma J, Yang B, Wang T, Zhu W and Tian J. 2019. Changes of alternative splicing in Arabidopsis thaliana grown under different $\mathrm{CO}_{2}$ concentrations. Gene 689:43-50. DOI 10.1016/j.gene.2018.11.083

Iida K. 2004. Genome-wide analysis of alternative pre-mRNA splicing in Arabidopsis thaliana based on fulllength cDNA sequences. Nucleic Acids Research 32:5096-5103. DOI 10.1093/nar/gkh845

Jiang LC, Zhang QX, Pu XB and Zhang ZY. 1998. Evaluation of Genetic and Physiological Characteristics of Aggregate-siliqua Rapeseed. Southwest China Journal of Agricultural Sciences 11(04): 62-68. (in Chinese)

Kanehisa M, Araki M, Goto S, Hattori M, Hirakawa M, Itoh M, Katayama T, Kawashima S, Okuda S, Tokimatsu T and Yamanishi Y. 2007. KEGG for linking genomes to life and the environment. Nucleic Acids Research 36:D480-D484. DOI 10.1093/nar/gkm882

Kim D, Pertea G, Trapnell C, Pimentel H, Kelley R and Salzberg SL. 2013. TopHat2: accurate alignment of transcriptomes in the presence of insertions, deletions and gene fusions. Genome Biology 14:R36. DOI 10.1186/gb-2013-14-4-r36

Labadorf A, Link A, Rogers MF, Thomas J, Reddy AS and Ben-Hur A. 2010. Genome-wide analysis of alternative splicing in Chlamydomonas reinhardtii. BMC Genomics 11:114. DOI 10.1186/1471-2164-11114

Leivar P and Monte E 2014. PIFs: systems integrators in plant development. Plant Cell 26:56-78. DOI $10.1105 /$ tpc. 113.120857

Li S, Chen L, Zhang L, Li X, Liu Y, Wu Z, Dong F, Wan L, Liu K and Hong D. 2015. BnaC9. SMG7b functions as a positive regulator of the number of seeds per silique in Brassica napus by regulating the

Peer] reviewing PDF | (2019:12:44287:4:0:NEW 6 Sep 2020) 
592

593

594

595

596

597

598

599

600

601

602

603

604

605

606

607

608

609

610

611

612

613

614

615

616

617

618

619

620

621

622

623

624

625

626

627

628

629

630

631

632

633

634

635

636

formation of functional female gametophytes. Plant Physiology 169:2744-2760. DOI

$10.1104 /$ pp. 15.01040

Li W, Zhang Q, Kong X, Wu C, Ma X, Zhang H and Zhao Y. 2009. Salt Tolerance is Conferred in Arabidopsis by Overexpression of the Vacuolar $\mathrm{Na}(+) / \mathrm{H}(+)$ Antiporter Gene $S s N H X 2$, an Alternative

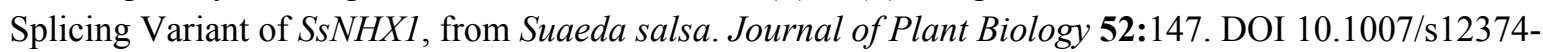
009-9016-Z

Liu H, Tan M, Yu H, Li L, Zhou F, Yang M, Zhou T and Zhao Y. 2016. Comparative transcriptome profiling of the fertile and sterile flower buds of a dominant genic male sterile line in sesame (Sesamum indicum L.). BMC Plant Biology 16:250. DOI 10.1186/s12870-016-0934-X

Liu J, Hua W, Hu Z, Yang H, Zhang L, Li R, Deng L, Sun X, Wang X and Wang H. 2015. Natural variation in ARF18 gene simultaneously affects seed weight and silique length in polyploid rapeseed. Proceedings of the National Academy of Sciences 112:E5123-E5132. DOI 10.1073/pnas.1502160112

Lolas IB, Himanen K, Grønlund JT, Lynggaard C, Houben A, Melzer M, Van Lijsebettens M and Grasser KD. 2010. The transcript elongation factor FACT affects Arabidopsis vegetative and reproductive development and genetically interacts with HUB1/2. The Plant Journal 61:686-697. DOI 10.1111/j.1365-313X.2009.04096.x

Mao X, Cai T, Olyarchuk JG and Wei L. 2005. Automated genome annotation and pathway identification using the KEGG Orthology (KO) as a controlled vocabulary. Bioinformatics 21:3787-3793. DOI 10.1093/bioinformatics/bti430

Marioni JC, Mason CE, Mane SM, Stephens M and Gilad Y. 2008. RNA-seq: An assessment of technical reproducibility and comparison with gene expression arrays. Genome Research 18:1509-1517. DOI $10.1101 /$ gr.079558.108

Mortazavi A, Williams BA, Mccue K, Schaeffer L and Wold B. 2008. Mapping and quantifying mammalian transcriptomes by RNA-Seq. Nature Methods 5:621-628. DOI 10.1038/nmeth.1226

Nadolska-Orczyk A, Rajchel IK, Orczyk W and Gasparis S. 2017. Major genes determining yield-related traits in wheat and barley. Theoretical and Applied Genetics 130:1081-1098. DOI 10.1007/s00122-0172880-X

Nishida S, Kakei Y, Shimada Y and Fujiwara T. 2017. Genome-wide analysis of specific alterations in transcript structure and accumulation caused by nutrient deficiencies in Arabidopsis thaliana. The Plant Journal 91:741-753. DOI 10.1111/tpj.13606

Oeffinger M, Dlakic $M$ and Tollervey D. 2004. A pre-ribosome-associated HEAT-repeat protein is required for export of both ribosomal subunits. Genes \& Development 18:196-209. DOI 10.1101/gad.285604

Ozsolak F and Milos PM. 2011. RNA sequencing: advances, challenges and opportunities. Nature Reviews Genetics 12:87-98. DOI 10.1038/nrg2934

Palusa SG, Ali GS and Reddy ASN. 2007. Alternative splicing of pre-mRNAs of Arabidopsis serine/arginine-rich proteins: regulation by hormones and stresses. The Plant Journal 49:1091-1107. DOI 10.1111/j.1365-313X.2006.03020.x

Pan Q, Shai O, Lee LJ, Frey BJ and Blencowe BJ. 2008. Deep surveying of alternative splicing complexity in the human transcriptome by high-throughput sequencing. Nature Genetics 40:1413-1415. DOI 10.1038/ng.259

Peng PF, Li YC, Mei DS, Colasanti J, Fu L, Liu J, Chen YF and Hu Q. 2015. Expression divergence of FRUITFULL homeologs enhanced pod shatter resistance in Brassica napus. Genetics and Molecular Research 14:871-885. DOI 10.4238/2015.February.2.11

Pirone C, Gurrieri L, Gaiba I, Adamiano A, Valle F, Trost P and Sparla F. 2017. The analysis of the different functions of starch-phosphorylating enzymes during the development of Arabidopsis thaliana

Peer] reviewing PDF | (2019:12:44287:4:0:NEW 6 Sep 2020) 
637

638

639

640

641

642

643

644

645

646

647

648

649

650

651

652

653

654

655

656

657

658

659

660

661

662

663

664

665

666

667

668

669

670

671

672

673

674

675

676

677

678

679

680

681

682

plants discloses an unexpected role for the cytosolic isoform GWD2. Physiologia Plantarum 160:447457. DOI 10.1111/ppl.12564

Reddy, A.S.N. 2007. Alternative Splicing of Pre-Messenger RNAs in Plants in the Genomic Era. Annual Review of Plant Biology 58:267-294. 10.1146/annurev.arplant.58.032806.103754

Ren X, Liu Y, Jeong HK, Soundararajan P and Jeong BR. 2019. Temperature affects morphology, physiology, and biochemistry of plug seedlings of Astragalus membranaceus. Acta Physiologiae Plantarum 41:9. DOI 10.1007/s11738-018-2799-0

Ruan Y, Xu S, White R and Furbank RT. 2004. Genotypic and Developmental Evidence for the Role of Plasmodesmatal Regulation in Cotton Fiber Elongation Mediated by Callose Turnover. Plant Physiology 136:4104. DOI 10.1104/pp.104.051540

Sablok G, Gupta PK, Baek JM, Vazquez F and Min XJ. 2011. Genome-wide survey of alternative splicing in the grass Brachypodium distachyon: a emerging model biosystem for plant functional genomics. Biotechnology Letters 33:629-636. DOI 10.1007/s10529-010-0475-6

Sarazin V, Duclercq J, Mendou B, Aubanelle L, Nicolas V, Aono M, Pilard S, Guerineau F, SangwanNorreel B and Sangwan R S. 2015. Arabidopsis BNT1, an atypical TIR-NBS-LRR gene, acting as a regulator of the hormonal response to stress. Plant Science 239:216-229. DOI 10.1016/j.plantsci.2015.07.017

Seo PJ, Park MJ and Park CM. 2013. Alternative splicing of transcription factors in plant responses to low temperature stress: mechanisms and functions. Planta 237:1415-1424. DOI 10.1007/s00425-013-1882-4

Seong ES, Yoo JH, Kim NJ, Choi JH, Lee JG, Ghimire BK, Chung IM and Yu CY. 2016. Morphological changes and increase of resistance to oxidative stress by overexpression of the LebZIP2 gene in Nicotiana benthamiana. Russian Journal of Plant Physiology 63:124-131. DOI 10.1134/S1021443716010143

Slotte T, Huang H, Holm K, Ceplitis A, Onge KS, Chen J, Lagercrantz U and Lascoux M. 2009. Splicing variation at a FLOWERING LOCUS C homeolog is associated with flowering time variation in the tetraploid Capsella bursa-pastoris. Genetics 183:337-345. DOI 10.1534/genetics.109.103705

Sultan M, Schulz MH, Richard H, Magen A, Klingenhoff A, Scherf M, Seifert M, Borodina T, Soldatov A, Parkhomchuk D, Schmidt D, O'Keeffe S, Haas S, Vingron M, Lehrach H and Yaspo ML. 2008. A global view of gene activity and alternative splicing by deep sequencing of the human transcriptome. Science 321:956-960. DOI 10.1126/science.1160342

Syed NH, Kalyna M, Marquez Y, Barta A and Brown JWS. 2012. Alternative splicing in plants - coming of age. Trends in Plant Science 17:616-623. DOI 10.1016/j.tplants.2012.06.001

Tamada Y, Nakamori K, Nakatani H, Matsuda K, Hata S, Furumoto T and Izui K. 2007. Temporary Expression of the TAF10 Gene and its Requirement for Normal Development of Arabidopsis thaliana. Plant and Cell Physiology 48:134-146. DOI 10.1093/pcp/pc1048

Tong C, Wang X, Yu J, Wu J, Li W, Huang J, Dong C, Hua W and Liu S. 2013. Comprehensive analysis of RNA-seq data reveals the complexity of the transcriptome in Brassica rapa. BMC Genomics 14:689. DOI 10.1186/1471-2164-14-689

Tuttle JR, Nah G, Duke MV, Alexander DC, Guan X, Song Q, Chen ZJ, Scheffler BE and Haigler CH. 2015. Metabolomic and transcriptomic insights into how cotton fiber transitions to secondary wall synthesis, represses lignification, and prolongs elongation. BMC Genomics 16:477. DOI 10.1186/s12864015-1708-9

Wang L, Wang W, Wang Y, Liu Y, Wang J, Zhang X, Ye D and Chen L. 2013. Arabidopsis Galacturonosyltransferase (GAUT) 13 and GAUT14 Have Redundant Functions in Pollen Tube Growth. Molecular Plant 6:1131-1148. DOI 10.1093/mp/sst084

Wang Z, Cao H, Sun Y, Li X, Chen F, Carles A, Li Y, Ding M, Zhang C, Deng X, Soppe WJ and Liu YX. 2013. Arabidopsis paired amphipathic helix proteins SNL1 and SNL2 redundantly regulate primary

Peer] reviewing PDF | (2019:12:44287:4:0:NEW 6 Sep 2020) 
683

684

685

686

687

688

689

690

691

692

693

694

695

696

697

698

699

700

701

702

703

704

705

706

707

708

709

710

711

712

713

714

715

716

717

718

719

720

721

722

723

724

725

726

727

728

seed dormancy via abscisic acid-ethylene antagonism mediated by histone deacetylation. Plant Cell 25:149-166. DOI 10.1105/tpc.112.108191

Wang Z, Chen F, Li X, Cao H, Ding M, Zhang C, Zuo J, Xu C, Xu J, Deng X, Xiang Y, Soppe WJJ and Liu Y. 2016. Arabidopsis seed germination speed is controlled by SNL histone deacetylase-binding factor-mediated regulation of AUX1. Nature Communications 7:13412. DOI 10.1038/ncomms 13412

Wang Z, Gerstein M, and Snyder M. 2009. RNA-Seq: a revolutionary tool for transcriptomics. Nature Reviews Genetics 10:57-63. DOI 10.1038/nrg2484

Xia T, Zhang L, Xu J, Wang L, Liu B, Hao M, Chang X, Zhang T, Li S, Zhang H, Liu D and Shen Y. 2017. The alternative splicing of EAM8 contributes to early flowering and short-season adaptation in a landrace barley from the Qinghai-Tibetan Plateau. Theoretical and Applied Genetics 130:757-766. DOI 10.1007/s00122-016-2848-2

Xiao L, Li X, Liu F, Zhao Z, Xu L, Chen C, Wang Y, Shang G and Du D. 2018. Mutations in the CDS and promoter of BjuA07.CLV1 cause a multilocular trait in Brassica juncea. Scientific Reports 8:5339. DOI 10.1038/s41598-018-23636-4

Xiao L, Zhao H, Zhao Z, Du D, Xu L, Yao Y, Zhao Z, Xing X, Shang G and Zhao H. 2013. Genetic and physical fine mapping of a multilocular gene Bjln1 in Brassica juncea to a 208-kb region. Molecular Breeding 32:373-383. DOI 10.1007/s11032-013-9877-1

Xun H, Yang X, He H, Wang M, Guo P, Wang Y, Pang J, Dong Y, Feng X, Wang S and Liu B. 2019. Over-expression of $G m K R 3$, a TIR-NBS-LRR type $R$ gene, confers resistance to multiple viruses in soybean. Plant Molecular Biology 99:95-111. DOI 10.1007/s11103-018-0804-z

Yadava SK, Paritosh K, Panjabi-Massand P, Gupta V, Chandra A, Sodhi YS, Pradhan AK and Pental D. 2014. Tetralocular ovary and high silique width in yellow sarson lines of Brassica rapa (subspecies trilocularis) are due to a mutation in Bra034340 gene, a homologue of CLAVATA3 in Arabidopsis. Theoretical and Applied Genetics 127:2359-2369. DOI 10.1007/s00122-014-2382-z

Yang Z, Chen Z, Peng Z, Yu Y, Liao M, Wei S. 2017. Development of a high-density linkage map and mapping of the three-pistil gene (Pis 1) in wheat using GBS markers. BMC Genomics 18:567. DOI 10.1186/s12864-017-3960-7

Ying S, Zhang D, Fu J, Shi Y, Song Y, Wang T and Li Y. 2012. Cloning and characterization of a maize bZIP transcription factor, ZmbZIP72, confers drought and salt tolerance in transgenic Arabidopsis. Planta 235:253-266. DOI 10.1007/s00425-011-1496-7

Young MD, Wakefield MJ, Smyth GK and Oshlack A. 2010. Gene ontology analysis for RNA-seq: accounting for selection bias. Genome Biology 11:R14. DOI 10.1186/gb-2010-11-2-r14

Yu C, Guo Y, Ge J, Hu Y, Dong J and Dong Z. 2015. Characterization of a new temperature-sensitive male sterile line SP2S in rapeseed (Brassica napus L.). Euphytica 206:473-485. DOI 10.1007/s10681-0151514-0

Yu J, Han J, Kim Y, Song M, Yang Z, He Y, Fu R, Luo Z, Hu J, Liang W and Zhang D. 2017. Two rice receptor-like kinases maintain male fertility under changing temperatures. Proceedings of the National Academy of Sciences 114:12327-12332. DOI 10.1073/pnas.1705189114

Zhang GQ, He Y, Xu L, Tang GX, Zhou WJ. 2006. Genetic analyses of agronomic and seed quality traits of doubled haploid population in Brassica napus through microspore culture. Euphytica 149:169-177. DOI 10.1007/s10681-005-9064-5

Zhu X, Ni Y, He R, Jiang Y, Li Q and Niu J. 2019. Genetic mapping and expressivity of a wheat multi-pistil gene in mutant 12TP. Journal of Integrative Agriculture 18:532-538. DOI 10.1016/S20953119(18)61935-5

Zou M, Guan Y, Ren H, Zhang F and Chen F. 2008. A bZIP transcription factor, OsABI5, is involved in rice fertility and stress tolerance. Plant Molecular Biology 66:675-683. DOI 10.1007/s11103-008-9298-4 
729

730

Peer) reviewing PDF | (2019:12:44287:4:0:NEW 6 Sep 2020) 
Figure 1

Multi-silique trait in zws-ms, compared with the single siliques of its near-isogenic line zws-217.

(A) Primary inflorescences. (B) Siliques. 


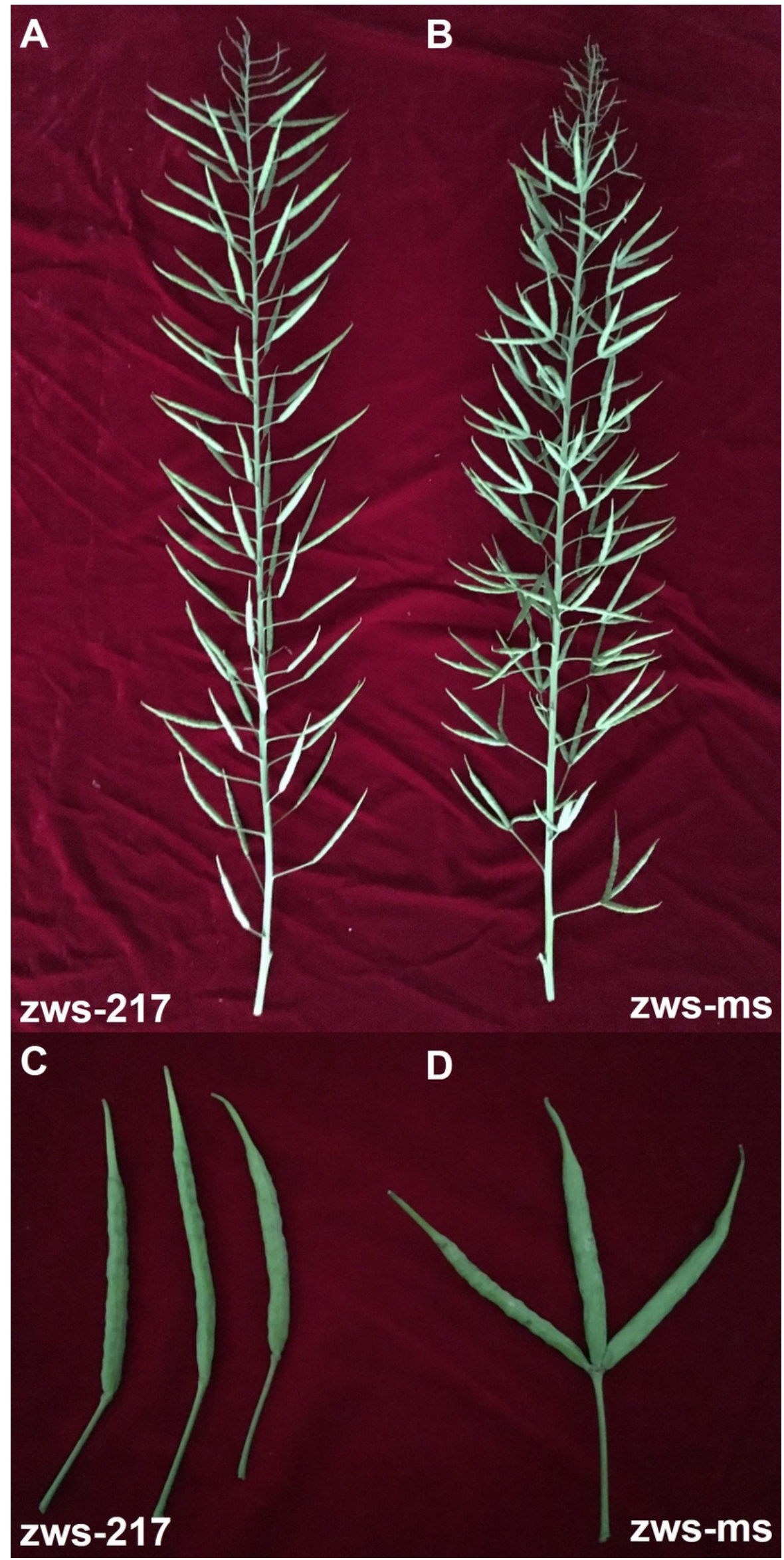

Peer] reviewing PDF | (2019:12:44287:4:0:NEW 6 Sep 2020) 
Figure 2

Statistics of different alternative splicing types of each line.

(A) data of plants grown in Xindu; (B) data from colder area Ma'erkang. horizontal axis shows the number of each AS type; vertical axis shows different types of AS events.

A

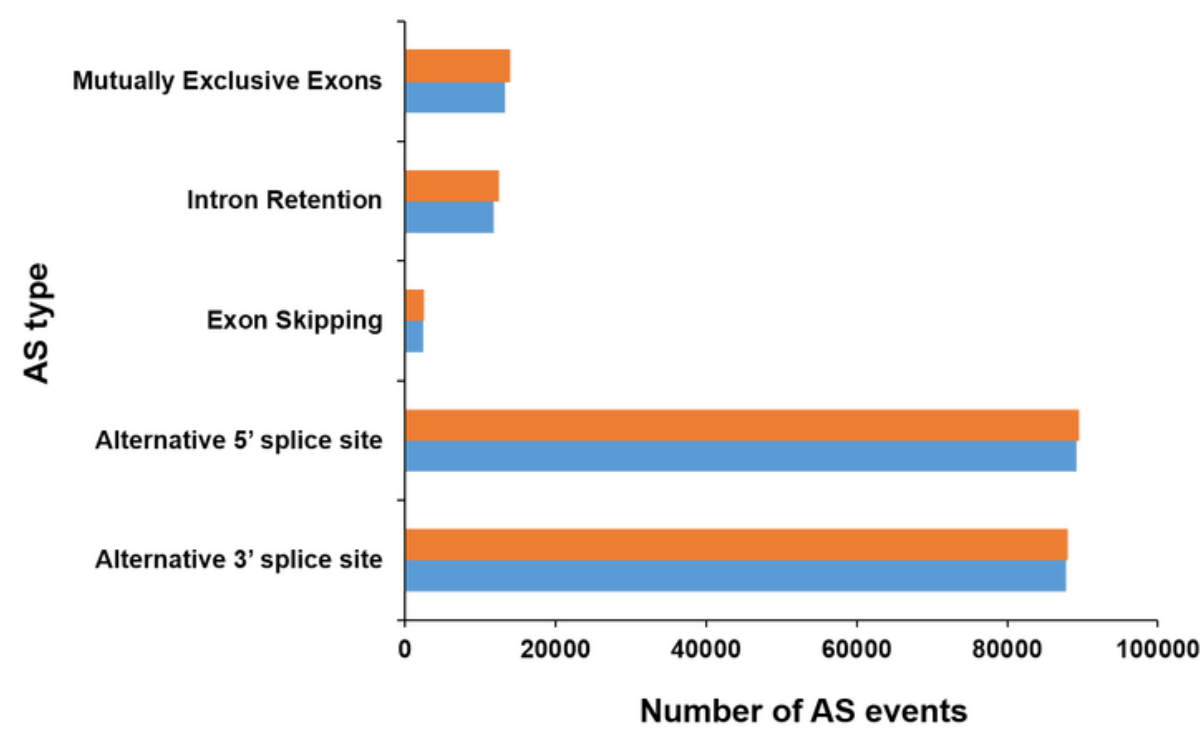

B

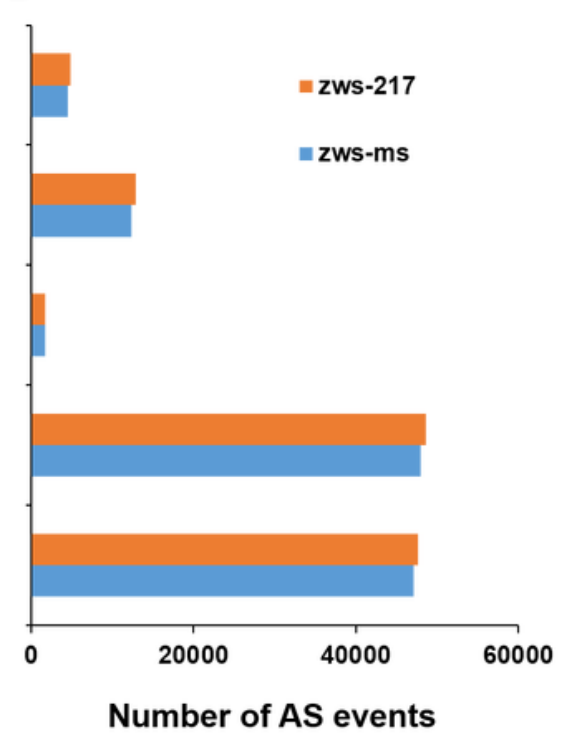




\section{Figure 3}

Gene ontology (GO) terms associated with the alternatively spliced genes.

GO terms were divided into three categories: biological processes, cellular components, and molecular functions. (A) data of plants grown in Xindu; (B) data from colder area Ma'erkang. Note: The x-axis shows the GO categories and subclasses of the alternatively spliced genes. the $y$-axis shows the number or percentage of annotated alternatively spliced genes.

A
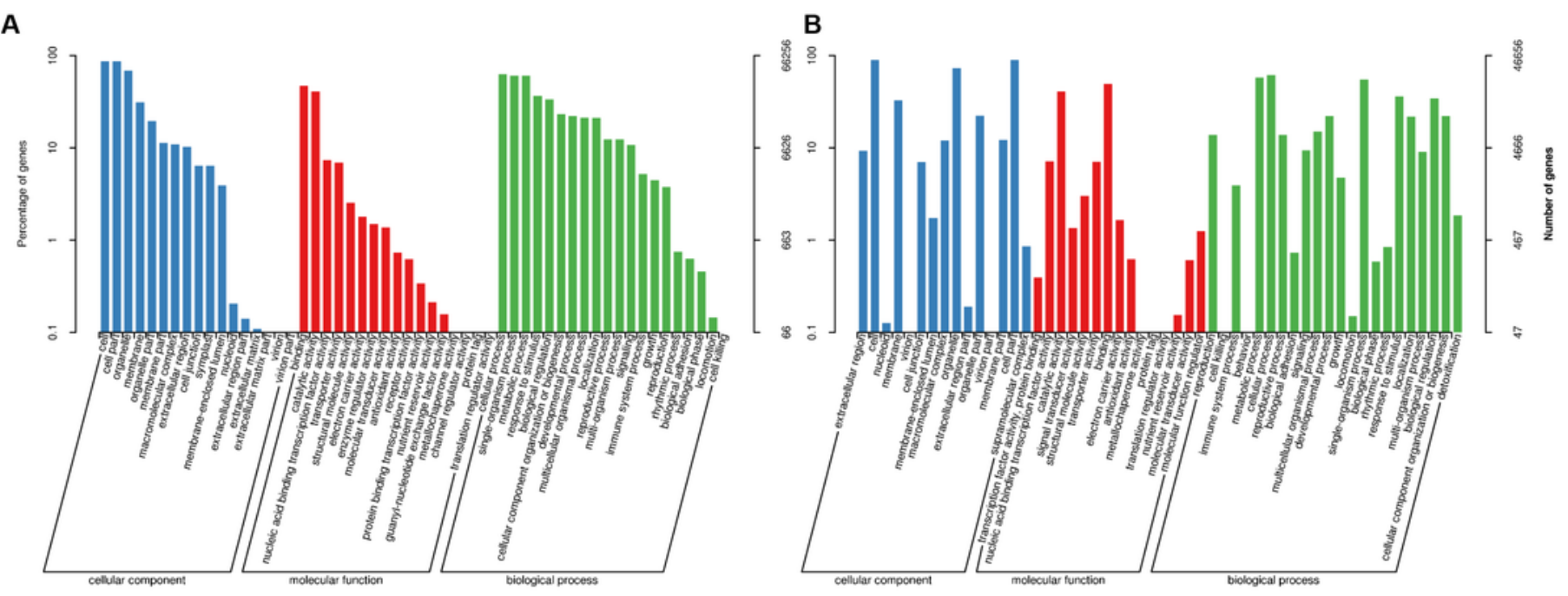
Figure 4

Classified KEGG pathways associated with the alternatively spliced genes.

The pathways were classified into five major groups: metabolism, genetic information processing, cellular processes, environmental information processing, and organismal systems. (A) data of plants grown in Xindu; (B) data from colder area Ma'erkang. Note: The Xaxis shows the number of annotated alternatively spliced genes; the $y$-axis shows the pathway categories.

A

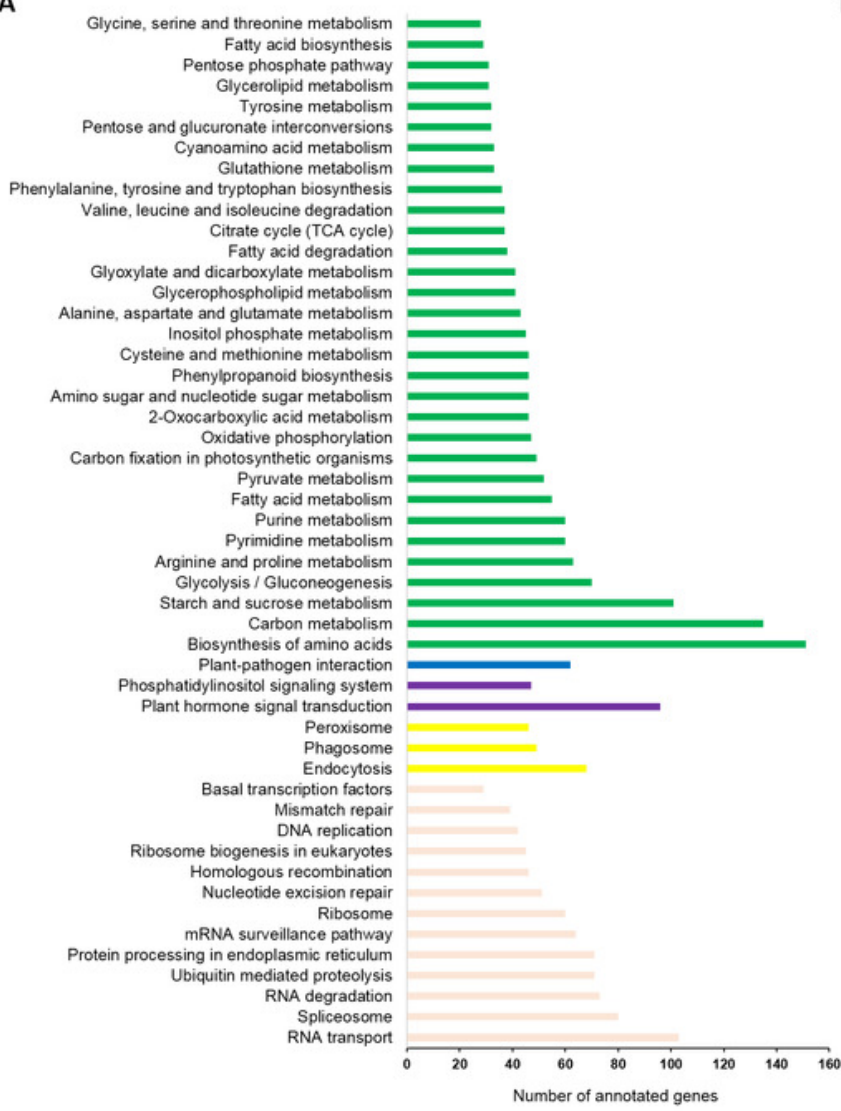

B

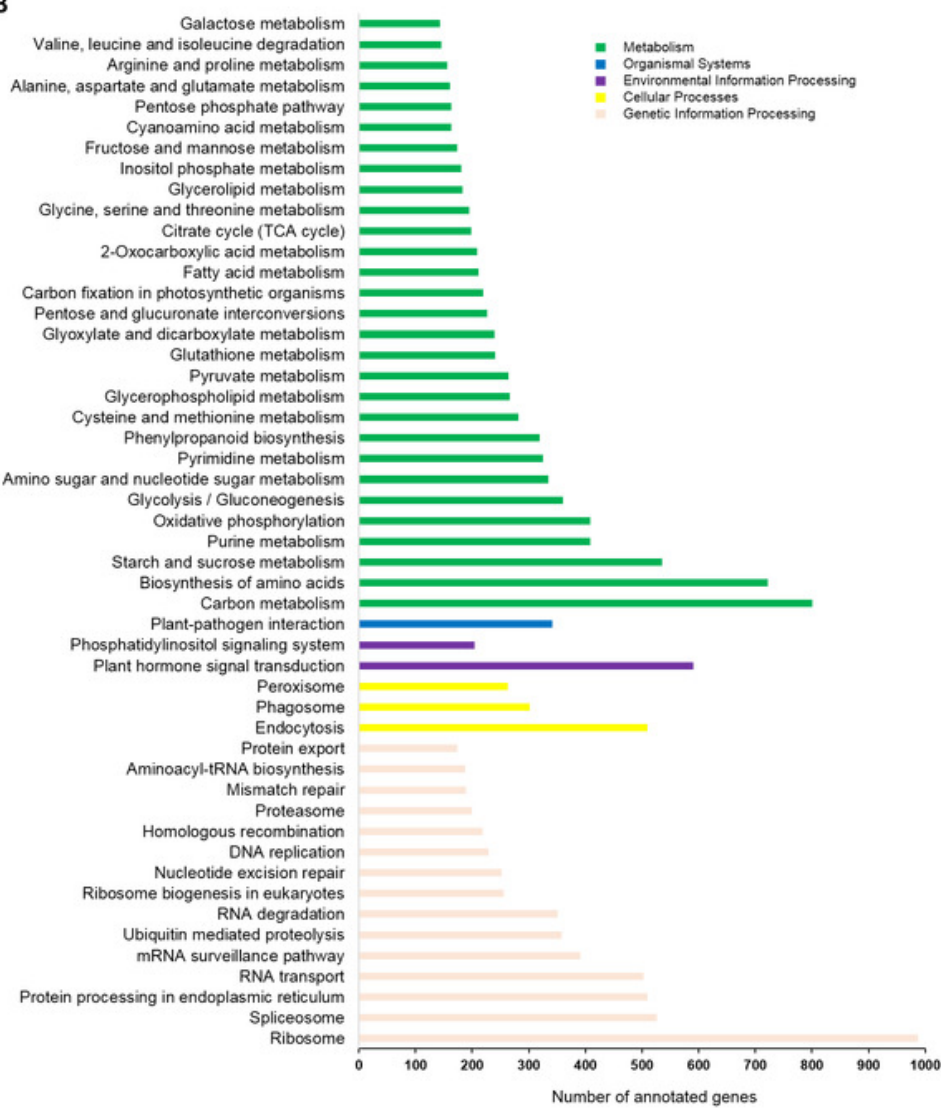




\section{Table $\mathbf{1}$ (on next page)}

Numbers of alternative splicing events in six samples.

Note: T01, T02, and T03: Buds of three independent zws-ms plants at the budding stage;

T04, T05, and T06: Buds of three independent zws-217 plants at the budding stage.

Alternative 3' splice site: different-size mRNAs are produced depending on the usage of a proximal or distal 3' splice site; Alternative 5' splice site: different-size mRNAs are produced depending on the use of a proximal or distal 5' splice site; Exon Skipping: an exon is either included or excluded from the mRNA; Intron Retention: an intron is either retained or excised in the mRNA, resulting in different-size transcripts; Mutually Exclusive Exons: adjacent exons are spliced in such a way that only one of them is included at a time in the mRNA. 
Table 1. Numbers of alternative splicing events in six samples.

\begin{tabular}{|c|c|c|c|c|c|c|c|}
\hline Area & Line & Sample ID & Alternative 3' splice site & Alternative 5' splice site & Exon Skipping & Intron Retention & Mutually Exclusive Exons \\
\hline \multirow{6}{*}{ Xindu } & \multirow{4}{*}{ zWs-ms } & T01 & 87699 & 89187 & 2333 & 11981 & 12765 \\
\hline & & T02 & 87603 & 88889 & 2404 & 11201 & 13100 \\
\hline & & T03 & 88055 & 89623 & 2638 & 12184 & 14060 \\
\hline & & T04 & 87953 & 89443 & 2567 & 12501 & 13887 \\
\hline & \multirow[t]{2}{*}{ zws-217 } & T05 & 88012 & 89588 & 2581 & 12722 & 13901 \\
\hline & & T06 & 88001 & 89408 & 2549 & 12373 & 13768 \\
\hline \multirow{6}{*}{$\begin{array}{l}\text { Ma'erkang } \\
\text { (colder area) }\end{array}$} & \multirow{3}{*}{ zWs-ms } & T01 & 48432 & 49501 & 1970 & 14764 & 5299 \\
\hline & & T02 & 46307 & 47031 & 1526 & 9560 & 3696 \\
\hline & & T03 & 46594 & 47558 & 1712 & 12686 & 4464 \\
\hline & \multirow{3}{*}{ zWs-217 } & T04 & 48204 & 49168 & 1596 & 12722 & 4733 \\
\hline & & T05 & 46902 & 47912 & 1754 & 12194 & 4777 \\
\hline & & T06 & 47759 & 48853 & 1822 & 13718 & 5105 \\
\hline
\end{tabular}

2 Note: T01, T02, and T03: Buds of three independent zws-ms plants at the budding stage; T04, T05, and T06: Buds of three independent zws-217 plants at 3 the budding stage.

4 Alternative 3' splice site: different-size mRNAs are produced depending on the usage of a proximal or distal 3' splice site; Alternative 5' splice site:

5 different-size mRNAs are produced depending on the use of a proximal or distal 5' splice site; Exon Skipping: an exon is either included or excluded from

6 the mRNA; Intron Retention: an intron is either retained or excised in the mRNA, resulting in different-size transcripts; Mutually Exclusive Exons: adjacent

7 exons are spliced in such a way that only one of them is included at a time in the mRNA. 
Table 2 (on next page)

Eleven differently expressed alternatively spliced genes and their annotations. 
Table 2. Eleven differently expressed alternatively spliced genes and their annotations.

\begin{tabular}{|c|c|c|c|c|c|}
\hline Gene ID & $\begin{array}{l}\text { Up- or down- } \\
\text { regulated }\end{array}$ & FDR & $\log _{2} \mathrm{FC}$ & GO annotation & KEGG annotation \\
\hline BnaA02g02630D & down & $1.53641 \mathrm{E}-06$ & -2.01793 & $\begin{array}{l}\text { Cellular Component: Golgi apparatus (GO:0005794); Biological Process: pollen development (GO:0009555); } \\
\text { Biological Process: pollen tube growth (GO:0009860); Molecular Function: polygalacturonate 4-alpha- } \\
\text { galacturonosyltransferase activity (GO:0047262); Biological Process: cell wall pectin biosynthetic process } \\
\text { (GO:0052325); Cellular Component: pollen tube (GO:0090406); }\end{array}$ & $\begin{array}{l}\text { K13648|0|brp:103850984|probable } \\
\text { galacturonosyltransferase 14; K13648 alp } \\
\text { galacturonosyltransferase [EC:2.4.1.43] }\end{array}$ \\
\hline BnaA02g03080D & down & $1.28889 \mathrm{E}-05$ & -2.07335 & -- & $\begin{array}{l}\text { K15203|1.23362e- } \\
59 \mid \text { brp: } 103851045 \mid \text { uncharacterized } \\
\text { LOC103851045; K15203 general transcr } \\
\text { factor 3C polypeptide } 6 \text { (A) }\end{array}$ \\
\hline BnaA04g16220D & down & $4.07836 \mathrm{E}-11$ & -2.10257 & -- & -- \\
\hline BnaA07g04500D & up & $1.84613 \mathrm{E}-33$ & 2.610402 & Cellular Component: mitochondrion (GO:0005739); & -- \\
\hline BnaA09g45000D & down & $1.26222 \mathrm{E}-12$ & -2.66637 & $\begin{array}{l}\text { Biological Process: RNA splicing, via endonucleolytic cleavage and ligation (GO:0000394); Molecular Function: } \\
\text { DNA binding (GO:0003677); Cellular Component: transcription factor TFIID complex (GO:0005669); Biological } \\
\text { Process: DNA-templated transcription, initiation (GO:0006352); Biological Process: transcription from RNA } \\
\text { polymerase II promoter (GO:0006366); Biological Process: cytokinin-activated signaling pathway (GO:0009736); } \\
\text { Biological Process: jasmonic acid mediated signaling pathway (GO:0009867); Biological Process: regulation of } \\
\text { ethylene-activated signaling pathway (GO:0010104); Molecular Function: protein heterodimerization activity } \\
\text { (GO:0046982); }\end{array}$ & $\begin{array}{l}\text { K03126|0|brp: } 103842750 \mid \text { transcription in } \\
\text { factor TFIID subunit } 12 \mathrm{~b}-\text { like; K03126 } \\
\text { transcription initiation factor TFIID subu } \\
\text { (A) }\end{array}$ \\
\hline BnaA09g45260D & down & $1.75043 \mathrm{E}-11$ & -2.14465 & $\begin{array}{l}\text { Cellular Component: chloroplast (GO:0009507); } \\
\text { Molecular Function: sequence-specific DNA binding transcription factor activity (GO:0003700); Cellular }\end{array}$ & -- \\
\hline BnaAnng30260D & up & $1.04924 \mathrm{E}-26$ & 4.006055 & $\begin{array}{l}\text { Component: nucleus (GO:0005634); Biological Process: response to xenobiotic stimulus (GO:0009410); Biological } \\
\text { Process: response to ethylene (GO:0009723); Biological Process: hormone-mediated signaling pathway } \\
\text { (GO:0009755); Biological Process: endoplasmic reticulum unfolded protein response (GO:0030968); Biological } \\
\text { Process: positive regulation of transcription, DNA-templated (GO:0045893); Molecular Function: protein } \\
\text { heterodimerization activity (GO:0046982); Biological Process: positive regulation of seed maturation } \\
\text { (GO:2000693); }\end{array}$ & -- \\
\hline
\end{tabular}




\begin{tabular}{|c|c|c|c|c|}
\hline BnaC02g06440D & down & $5.63055 \mathrm{E}-15$ & -2.04283 & Cellular Component: cytoplasm (GO:0005737); \\
\hline \multirow[t]{3}{*}{ BnaC06g16950D } & up & 4.45014E-05 & 3.19811 & -- \\
\hline & & & & Molecular Function: protein binding (GO:0005515); Cellular Component: nucleus (GO:0005634); Cellular \\
\hline & & & & Component: mitochondrion (GO:0005739); Cellular Component: cytosol (GO:0005829); Biological Process: \\
\hline \multirow[t]{6}{*}{ BnaC07g33980D } & up & 2.15674E-13 & 2.904839 & response to auxin (GO:0009733); Biological Process: systemic acquired resistance, salicylic acid mediated signaling \\
\hline & & & & pathway (GO:0009862); Biological Process: defense response to bacterium (GO:0042742); Molecular Function: \\
\hline & & & & ADP binding (GO:0043531); \\
\hline & & & & Molecular Function: protein binding (GO:0005515); Molecular Function: ATP binding (GO:0005524); Cellular \\
\hline & & & & Component: mitochondrion (GO:0005739); Biological Process: starch catabolic process (GO:0005983); Biological \\
\hline & & & & Process: circadian rhythm (GO:0007623); Cellular Component: chloroplast stroma (GO:0009570); Biological \\
\hline \multirow[t]{4}{*}{ BnaC08g49610D } & down & $1.65462 \mathrm{E}-10$ & -8.08437 & Process: response to symbiotic fungus (GO:0009610); Biological Process: cold acclimation (GO:0009631); Cellular \\
\hline & & & & Component: chloroplast envelope (GO:0009941); Biological Process: phosphorylation (GO:0016310); Biological \\
\hline & & & & Process: starch biosynthetic process (GO:0019252); Molecular Function: alpha-glucan, water dikinase activity \\
\hline & & & & (GO:0050521); \\
\hline
\end{tabular}

$-$

Molecular Function: protein binding (GO:0005515); Cellular Component: nucleus (GO:0005634); Cellular Component: mitochondrion (GO:0005739); Cellular Component: cytosol (GO:0005829); Biological Process: pathway (GO:0009862); Biological Process: defense response to bacterium (GO:0042742); Molecular Function: ADP binding (GO:0043531);

(GO:0050521);

K08244|0|brp:103843262|alpha-glucan w dikinase 1, chloroplastic; K08244 alphawater dikinase [EC:2.7.9.4] (A) 
Table 3 (on next page)

The 11 differently expressed alternatively spliced genes and their orthologs in Arabidopsis 
Table 3. The 11 differently expressed alternatively spliced genes and their orthologs in Arabidopsis

\begin{tabular}{lcc}
\hline Gene in B. napus & \multicolumn{2}{c}{ Orthologs in Arabidopsis } \\
\cline { 2 - 3 } BnaA02g02630D & AT5G15470 & Description \\
BnaA02g03080D & AT3G15420 & GALACTURONOSYLTRANSFERASE 14 (GAUT14) \\
BnaA04g16220D & AT1G14800 & Transcription factor TFIIIC, Tau55-related protein \\
BnaA07g04500D & AT2G04900 & Nucleic acid-binding, OB-fold-like protein \\
BnaA09g45000D & AT3G10070 & Unknown protein \\
BnaA09g45260D & AT1G15060 & TBP-ASSOCIATED FACTOR 12 (TAF12) \\
BnaAnng30260D & AT3G54620 & Uncharacterized conserved protein UCP031088, alpha/beta hydrolase \\
BnaC02g06440D & AT5G16210 & BASIC LEUCINE ZIPPER 25 (BZIP25) \\
BnaC06g16950D & AT3G59000 & HEAT repeat-containing protein \\
BnaC07g33980D & AT4G16900 & F-box/RNI-like superfamily protein \\
BnaC08g49610D & AT1G10760 & Disease resistance protein (TIR-NBS-LRR class) family \\
\hline
\end{tabular}




\section{Table 4 (on next page)}

The eight important candidate genes with line-specific alternative splicing events and their orthologs in Arabidopsis 
Table 4. The eight important candidate genes with line-specific alternative splicing events and their orthologs in

\section{Arabidopsis}

3

\begin{tabular}{cll}
\hline \multirow{2}{*}{ Gene in B. napus } & \multicolumn{1}{c}{ Orthologs in Arabidopsis } \\
\cline { 2 - 3 } BnaC06g32640D & Gene ID & \multicolumn{1}{c}{ Description } \\
AT1G71692 & $\begin{array}{l}\text { AGAMOUS-LIKE 12 (AGL12) } \\
\text { PHYTOCHROME INTERACTING FACTOR 3-LIKE 5 (PIL5), PHY-INTERACTING FACTOR 1 } \\
\text { (PIF1) }\end{array}$ \\
BnaC04g31460D & AT2G20180 & Protein prenylyltransferase superfamily protein \\
BnaC05g34570D & AT5G17270 & P-loop containing nucleoside triphosphate hydrolases superfamily protein \\
BnaC04g26180D & AT3G18600 & GLUTATHIONE REDUCTASE (GR), EMB2360 \\
BnaC07g25280D & AT3G54660 & HIGH MOBILITY GROUP (HMG), NUCLEOSOME/CHROMATIN ASSEMBLY FACTOR D \\
BnaC03g32190D & AT3G28730 & (NFD), SSRP1 \\
BnaCnng68400D & AT4G00050 & UNFERTILIZED EMBRYO SAC 10 (UNE10), PIF8 \\
\hline
\end{tabular}

